Compound-Nuclear Reaction Cross Sections from Surrogate Measurements: Status and Challenges

J. Escher

February 29, 2008

Compound-Nuclear Reactions and Related Topics 2007 Fish Camp, CA, United States October 22, 2007 through October 26, 2007 
This document was prepared as an account of work sponsored by an agency of the United States government. Neither the United States government nor Lawrence Livermore National Security, LLC, nor any of their employees makes any warranty, expressed or implied, or assumes any legal liability or responsibility for the accuracy, completeness, or usefulness of any information, apparatus, product, or process disclosed, or represents that its use would not infringe privately owned rights. Reference herein to any specific commercial product, process, or service by trade name, trademark, manufacturer, or otherwise does not necessarily constitute or imply its endorsement, recommendation, or favoring by the United States government or Lawrence Livermore National Security, LLC. The views and opinions of authors expressed herein do not necessarily state or reflect those of the United States government or Lawrence Livermore National Security, LLC, and shall not be used for advertising or product endorsement purposes. 


\title{
Compound-Nuclear Reaction Cross Sections from Surrogate Measurements: Status and Challenges
}

\author{
Jutta Escher
}

Lawrence Livermore National Laboratory, Livermore, CA, USA

\begin{abstract}
The Surrogate nuclear reactions method, an indirect approach for determining cross sections for compoundnuclear reactions involving difficult-to-produce targets, is reviewed. The underlying formalism is outlined, the challenges involved in carrying out a complete Surrogate treatment are detailed, and the present status of the theory is summarized. The approximations employed in the analyses of Surrogate experiments are discussed and their validity is examined.
\end{abstract}

Keywords: compound nucleus, Surrogate reaction, indirect measurement, neutron-induced fission, radiative neutron capture PACS: $24.87 .+\mathrm{y}, 24.60 . \mathrm{Dr}, 24.50 .+\mathrm{g}, 25.85 . \mathrm{Ec}, 25.40 . \mathrm{Lw}$

\section{INTRODUCTION}

Compound-nuclear reactions play a crucial role in many areas of basic and applied nuclear science. The production of heavy elements in various astrophysical environments, for instance, involves compound reactions and the resulting observable abundance patterns depend, sometimes very sensitively, on the associated reaction cross sections. Similarly, a proper description of nuclear fuel cycles for energy applications requires data on various types of compound reactions.

The appropriate formalism for the description of compound-nuclear reactions is a statistical one [1]. Based on Bohr's hypothesis of the independence of formation and decay of the compound nucleus, the cross sections are calculated in the framework of the HauserFeshbach formalism, which properly takes account of the conservation of angular momentum and parity in the reaction. Under certain circumstances, the decay of the intermediate equilibrated system, the compound nucleus $(\mathrm{CN})$, becomes independent of its angular momentum and parity, and the cross section for the reaction factorizes into a simple product of a formation cross section and a decay probability for the exit channel of interest. This is the so-called "Weisskopf-Ewing" limit [2]. In other situations, corrections to the Hauser-Feshbach expressions are required, since the reaction of interest does not proceed through a completely equilibrated intermediate nuclear system. In this limit, pre-compound reactions begin to contribute to the cross section of interest $[2,3]$.

The independence of the formation and decay of a $\mathrm{CN}$ can be exploited to determine compound-nuclear reaction cross sections indirectly, via the so-called Surrogate nuclear reactions approach. In this approach the $\mathrm{CN}$ $\left(B^{*}\right)$ occurring in the reaction of interest $\left(a+A \rightarrow B^{*} \rightarrow\right.$ $c+C)$ is produced via an alternative, "Surrogate" reaction $\left(d+D \rightarrow B^{*}+b\right)$ and the measured $\mathrm{CN}$ decay proba- bilities are combined with the calculated formation cross section for the $\mathrm{CN}$ in the desired reaction to yield the relevant reaction cross section. This approach is particularly valuable when the target of interest, $A$, is short-lived and a suitable Surrogate reaction involving a stable target $D$ and a stable projectile $d$ can be identified.

The Surrogate approach was originally introduced in the 1970 s $[4,5]$ and has recently received renewed attention [6]-[17]. Both the early and the more recent applications of the method have focused primarily on cross section estimates for neutron-induced fission for actinide targets, although a few experiments have been designed to obtain $(\mathrm{n}, \gamma)$ cross sections for some rare earth and actinide nuclei. Almost all applications to date employ significant approximations in the determination of cross sections from Surrogate measurements. As applications to new areas of interest (lower energies, various types of $\mathrm{CN}$ reactions, new regions of the isotopic chart) are being explored, a more comprehensive treatment of the Surrogate approach becomes necessary in order to examine previously-used approximations, to validate the Surrogate approach, and to determine its limitations.

In this contribution, we review the Surrogate formalism, discuss the primary challenges to be addressed in order to obtain a comprehensive theoretical description of the Surrogate approach, and summarize the present status of the theory. We outline the approximation schemes currently employed in the analyses of Surrogate experiments, and discuss their validity.

\section{SURROGATE FORMALISM}

The Surrogate nuclear reaction technique combines experiment with theory to obtain cross sections for $\mathrm{CN}$ reactions, $a+A \rightarrow B^{*} \rightarrow c+C$, involving difficultto-produce targets, $A$. In the Hauser-Feshbach formal- 
ism [18], the cross section for this "desired" reaction takes the form:

$$
\sigma_{\alpha \chi}\left(E_{a}\right)=\sum_{J, \pi} \sigma_{\alpha}^{C N}\left(E_{e x}, J, \pi\right) G_{\chi}^{C N}\left(E_{e x}, J, \pi\right),
$$

with $\alpha$ and $\chi$ denoting the relevant entrance and exit channels, $a+A$ and $c+C$, respectively. The excitation energy $E_{e x}$ of the compound nucleus, $B^{*}$, is related to the center-of-mass energy $E_{a}$ in the entrance channel via the energy needed for separating $a$ from $B: E_{a}=$ $E-S_{a}(B)$. In many cases the formation cross section $\sigma_{\alpha}^{C N}=\sigma\left(a+A \rightarrow B^{*}\right)$ can be calculated to a reasonable accuracy by using optical potentials, while the theoretical decay probabilities $G_{\chi}^{C N}$ for the different decay channels $\chi$ are often quite uncertain. The latter are difficult to calculate accurately since they require knowledge of optical models, level densities, and strength functions for the various possible exit channels. The objective of the Surrogate method is to determine or constrain these decay probabilities experimentally.
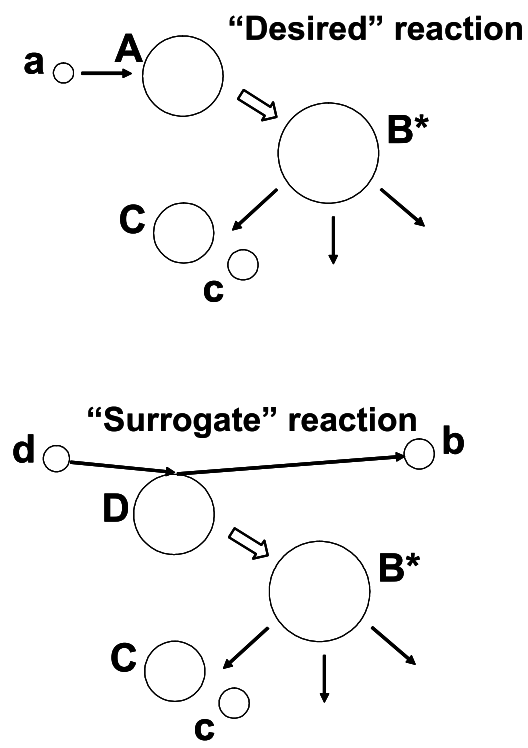

FIGURE 1. Schematic representation of the "desired" (top) and "Surrogate" (bottom) reaction mechanisms. The basic idea of the Surrogate approach is to replace the first step of the desired reaction, $a+A$, by an alternative (Surrogate) reaction, $d+D \rightarrow b+B^{*}$, that populates the same compound nucleus. The subsequent decay of the compound nucleus into the relevant channel, $c+C$, can then be measured and used to extract the desired cross section.

In the Surrogate approach, the compound nucleus $B^{*}$ is produced by means of an alternative ("Surrogate"), direct reaction, $d+D \rightarrow b+B^{*}$, and the desired decay channel $\chi\left(B^{*} \rightarrow c+C\right)$ is observed in coincidence with the outgoing particle $b$ (see Fig. 1). The coincidence measurement provides

$$
P_{\delta \chi}\left(E_{e x}\right)=\sum_{J, \pi} F_{\delta}^{C N}\left(E_{e x}, J, \pi\right) G_{\chi}^{C N}\left(E_{e x}, J, \pi\right)
$$

the probability that the $\mathrm{CN}$ was formed in the Surrogate reaction with spin-parity distribution $F_{\delta}^{C N}\left(E_{e x}, J, \pi\right)$ and subsequently decayed into the channel $\chi$. The distribution $F_{\delta}^{C N}\left(E_{e x}, J, \pi\right)$, which may be very different from the $\mathrm{CN}$ spin-parity populations following the absorption of the projectile $a$ in the desired reaction, has to be determined theoretically, so that the branching ratios $G_{\chi}^{C N}\left(E_{e x}, J, \pi\right)$ can be extracted from the measurements. In practice, the decay of the $\mathrm{CN}$ is modeled and the $G_{\chi}^{C N}\left(E_{e x}, J, \pi\right)$ are obtained by adjusting parameters in the model to reproduce the measured probabilities $P_{\delta \chi}\left(E_{e x}\right)[6,7]$. Subsequently, the sought-after cross section can be obtained by combining the calculated cross section $\sigma_{\alpha}^{C N}\left(E_{e x}, J, \pi\right)$ for the formation of $B^{*}$ (from $a+A$ ) with the extracted decay probabilities $G_{\chi}^{C N}\left(E_{e x}, J, \pi\right)$ for this state, see Eq. (1).

\section{SPIN-PARITY MISMATCH AND RELATED CHALLENGES}

The most significant limitation of the method at this time is the fact that the Surrogate reaction populates the states in the intermediate nucleus differently than the desired channel, i.e. the weights $F_{\delta}^{C N}\left(E_{e x}, J, \pi\right)$ by which the decay probabilities $G_{\chi}^{C N}\left(E_{e x}, J, \pi\right)$ are multiplied in Eq. (2) are different from the relative formation cross sections $f_{\alpha}^{C N}\left(E_{e x}, J, \pi\right)=\sigma_{\alpha}^{C N}\left(E_{e x}, J, \pi\right) / \sum_{J^{\prime} \pi^{\prime}} \sigma_{\alpha}^{C N}\left(E_{e x}, J^{\prime}, \pi^{\prime}\right)$ of Eq. (1), and depend on the direct reaction under consideration. This diffference is often referred to as the spinparity population mismatch.

Currently, our understanding of the spin-parity mismatch and its effect on cross sections extracted from Surrogate experiments is quite incomplete. When opticalmodel potentials are available, one can obtain fairly reliable spin-parity information for the desired reaction from optical-model calculations. An example is shown in Fig. 2. Plotted are spin populations of the the compound nucleus ${ }^{236} \mathrm{U}$, produced via neutron-absorption on ${ }^{235} \mathrm{U}$, for four different energies of the incoming neutron. However, no tools are currently available for formulating accurate predictions of the spin-parity distributions for compound nuclei produced in Surrogate reactions. This situation does not merely reflect an absence of useful reaction codes, but points to an incomplete picture of the reaction mechanisms that produce the compound nucleus in a Surrogate reaction.

Predicting the spin-parity distribution for a compound nucleus produced in a Surrogate reaction requires a careful consideration of the reaction mechanisms that are involved in the formation of the compound nucleus. In the absence of width fluctuation corrections, the challenge of describing the relevant reaction mechanisms can be 


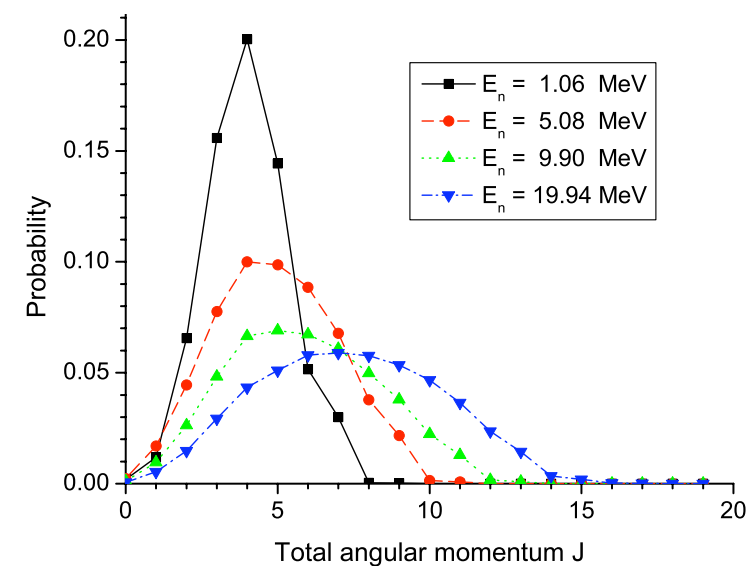

FIGURE 2. Angular-momentum distribution of positiveparity states of the compound nucleus ${ }^{236} \mathrm{U}$, following neutron absorption by ${ }^{235} \mathrm{U}$, for various neutron energies. The results, taken from Ref. [15], were obtained with Dietrich's optical potential FLAP [11]. The negative-parity distribution is not shown here as it is qualitatively similar.

divided into two separate problems:

1) the formation of a highly-excited nucleus in a direct reaction, and

2) the damping of the excited states into the compound nucleus.

The separation of the Surrogate reaction into two separate sub-processes is somewhat artificial, but may be useful conceptually. The Surrogate reaction is viewed as a mechanism that produces initially a highly-excited intermediate system. The system might consist, for instance, of a nucleon $N$ (stripped from the projectile $d$ in the reaction $d+D \rightarrow b+B^{*}$ ) plus the Surrogate target nucleus $D$. For the Surrogate approach to be valid, the $D+N$ system must subsequently fuse to produce the compound nucleus $B^{*}$, the decay of which one is interested in measuring. Decay of the intermediate system $(D+N$ in the example) by particle emission prior to reaching the equilibrated stage would invalidate the Surrogate approach, since the measured coincidence probabilities would no longer be associated with the decay of the compound nucleus of interest, $B^{*}$. It is thus important to understand how the configurations that are produced in the initial step evolve. Specifically, one needs to determine the probability for forming the desired compound nucleus $B^{*}$.

The above considerations do not include correlations between the incident and outgoing reaction channels, which in principle affect both the desired and Surrogate reactions. For the desired reaction, Eq. (1), these correlations can be taken into account formally by including width fluctuation corrections [2], while a similar simple solution is not readily available for the Hauser-
Feshbach-type expression describing the Surrogate reaction, Eq. (2). Therefore, a comprehensive theoretical treatment of the Surrogate method also requires an assessment of the importance of

3) width fluctuation correlations to the Surrogate reaction formalism and possibly an extension of the formalism to account for these correlations.

As the above issues have not yet been sufficiently addressed, one has to rely on approximations. Specifically, almost all applications of the Surrogate method so far have neglected the effects of the spin-parity mismatch and have analyzed Surrogate data under the assumption that the Weisskopf-Ewing approximation or a variant thereof, the Surrogate Ratio method, is valid. These approximations will be discussed below.

\section{ADDRESSING THE THEORY CHALLENGES}

In this section, we summarize some of the work done and outline further steps to be undertaken in order to address the challenges discussed above.

\section{Direct reaction to the continuum}

Addressing the first problem necessitates developing a quantitative description of the direct-reaction process that allows for a prediction of the spin-parity distribution in the highly-excited intermediate nucleus, immediately following the direct reaction. Such a description is nontrivial since it requires a framework for calculating cross sections of different reactions (stripping, pick-up, charge exchange, and inelastic scattering) to continuum states, for a variety of projectiles $(p, d, t, \alpha$, etc.) and targets (spherical, deformed, and transitional).

First steps towards predicting the spin-parity population following the initial step of a Surrogate reaction were taken by Andersen et al. [19], Back et al. [20], and, more recently, by Younes and Britt [6, 7]. These authors employed simple transfer calculations to estimate compound-nucleus spin-parity distributions following various stripping reactions on actinide targets. They neglected the possibility that the intermediate nucleus might decay prior to reaching equilibrium and took the resulting spin-parity distributions to be representative of those present in the compound nucleus created in the Surrogate reaction of interest. Younes and Britt used the calculated distributions to re-analyze Surrogate (t,pf), $\left({ }^{3} \mathrm{He}, \mathrm{df}\right)$, and $\left({ }^{3} \mathrm{He}, \mathrm{tf}\right)$ fission-correlation measurements from the 1970s $[4,5]$ in order to extract $(n, f)$ cross sections. Compared to earlier Surrogate analyses of the data, 
which ignored spin effects, their estimated (n,f) cross sections showed significantly improved agreement with evaluated results, where available. Their findings underscore the importance of accounting for the spin-parity mismatch between the desired and Surrogate reactions.

Inelastic scattering is potentially an important Surrogate mechanism for determining cross sections relevant to the astrophysical s process: $(n, \gamma)$ reactions on unstable s-process branch-point nuclei proceed through compound states of nuclei with stable ground states. Consequently, the compound states of interest can in principle be produced via an inelastic scattering reaction on a stable target. In Ref. [21], a simple model for predicting the spin-parity distribution of ${ }^{90} \mathrm{Zr}$, produced via inelastic $\alpha$ scattering, was developed. The model was based on the assumption that the inelastic scattering cross section for a (near-)spherical nucleus can be approximately expressed in terms of cross sections for producing uncorrelated particle-hole excitations in the target nucleus. Specifically, the cross section was given as an incoherent sum of DWBA scattering cross sections $\left(\frac{d \sigma}{d \Omega}\right)_{m_{h}, m_{p}}$ for individual particle-hole excitations $\left(m_{h}, m_{p}\right)$ with weights that depend on the energy and spreading widths of the particle-hole configurations. The calculated probabilities for populating different $J \pi$ states in the intermediate system are shown in Fig. 3, as a function of the scattering angle. The probabilities have been smoothed in order to account for experimental uncertainties and "binning" in the angular measurements. The distance between two adjacent curves gives the probability of finding the spin and parity indicated by the values listed at the right end of the upper curve. For example, at $90^{\circ}$, we find about $0 \%$ contribution from $0^{+}$states, $15 \%$ from $2^{+}$states, $8 \%$ from $4^{+}$states, etc. Information on such $J \pi$ distributions, their angular and energy dependence, is important for planning and interpreting Surrogate experiments.

\section{Damping into the compound nucleus}

The second problem to be addressed is associated with the evolution of the highly-excited intermediate system that is created in the initial stage of the Surrogate reaction. The assumption that a compound (i.e. equilibrated) nucleus is formed is central to the Surrogate method. Rapid decay of the intermediate configuration before a compound nucleus can be formed, which would invalidate the Surrogate analysis, needs to be excluded experimentally, or accounted for theoretically ${ }^{1}$.

\footnotetext{
${ }^{1}$ This process should not be confused with pre-equilibrium emission of particles in the desired reaction, $a+A \rightarrow c+C$; contributions from the latter cannot be determined via the Surrogate approach and need to be calculated separately and added to the desired cross section.
}

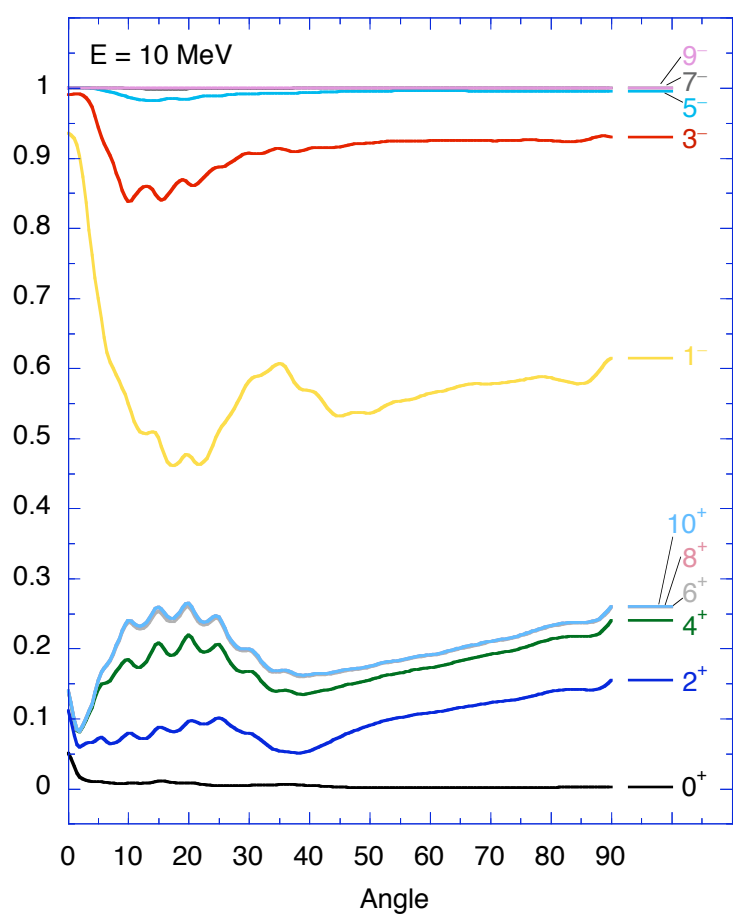

FIGURE 3. Probabilities for populating various $J \pi$ states in ${ }^{90} \mathrm{Zr}^{*}$ following inelastic alpha scattering with $140 \mathrm{MeV}$ projectiles that leave the target nucleus at excitation energy $E_{e x}=10 \mathrm{MeV}$. The probabilities have been smoothed in order to account for experimental uncertainties and "binning" in the angular measurements. The $J \pi$ distribution are seen to depend on the angle of the outgoing $\alpha$ particle, with the largest uncertainties occurring at forward angles $\left(<40^{\circ}\right)$.

New theory development is needed in order to determine to what extent those configurations that are produced in the initial step of a Surrogate reaction damp into a compound state. The competition between particle emission and equilibration is expected to not only depend on the direct reaction and energy chosen, but also on the spin and parity of the intermediate nucleus.

First steps towards understanding the evolution of the intermediate, highly-excited, system following a direct reaction have been taken by F.S. Dietrich. In Ref. [22], he considered the case of direct-semidirect neutron capture, employing the formalism developed by Parker et al. [23]. While direct (or direct-semidirect) nucleon capture is not typically considered in practical applications of the Surrogate method, the insights gained from studying this process have implications for the Surrogate approach. In particular, there is an obvious connection between direct radiative neutron capture to a highly-excited region of the intermediate nucleus and the deposition of a neutron to that region via a direct $(\mathrm{d}, \mathrm{p})$ reaction. In the former case, the energy of the $\gamma$-ray determines the excitation energy 
of the intermediate system, while in the latter case, the outgoing proton can be used to tag the energy of the nucleus. The formalism developed in Ref. [23] allows one to calculate the cross sections for both capture to unbound states (and thus for escape of the nucleon from the combined target-plus-nucleon system immediately after capture) and capture followed by absorption via an imaginary potential (which represents the formation of a compound nucleus). In Ref. [22], Dietrich studies the evolution of the ${ }^{90} \mathrm{Y}^{*}$ system following radiative capture of 19.6 MeV neutrons on ${ }^{89} \mathrm{Y}$. He finds that the reaction fails to form a compound nucleus at the $10 \%-15 \%$ level. Furthermore, he demonstrates that the probability for forming a compound nucleus depends on both the energy of the intermediate system, and on the angular-momentum distribution present following the direct reaction. These findings underscore the need to carefully study the competition between particle emission and equilibration following other direct reactions, in particular one-neutron stripping reactions, such as (d,p).

\section{Width fluctuation corrections}

Incorporating width fluctuation correlations will introduce additional complications. In the desired reaction, they are known to enhance the elastic scattering cross section and reduce the inelastic and reaction cross sections, although this depletion rarely exceeds $10-20 \%$ (even at energies below approximately $2 \mathrm{MeV}$ ) and becomes negligible as the excitation energy of the compound nucleus increases [2]. An examination of the role of width fluctuation correlations for Surrogate reactions has not yet been undertaken, but should be part of a comprehensive investigation of the formalism associated with the Surrogate approach.

\section{APPROXIMATIONS TO THE SURROGATE FORMALISM}

Here, we briefly review the approximations employed in current applications of the Surrogate method and summarize what is known about their validity.

\section{Weisskopf-Ewing approximation}

In the Weisskopf-Ewing approximation [2, 24] to the full Hauser-Feshbach formalism, the compound-nuclear decay probabilities are treated as independent of $J$ and $\pi$, and the cross section for the desired reaction takes the simple product form:

$$
\sigma_{\alpha \chi}^{W E}\left(E_{a}\right)=\sigma_{\alpha}^{C N}\left(E_{e x}\right) \mathscr{G}_{\chi}^{C N}\left(E_{e x}\right)
$$

where $\sigma_{\alpha}^{C N}\left(E_{e x}\right)=\sum_{J, \pi} \sigma_{\alpha}^{C N}\left(E_{e x}, J, \pi\right)$ is the reaction cross section describing the formation of the compound nucleus in the desired reaction and $\mathscr{G}_{\chi}^{C N}\left(E_{e x}\right)$ denotes the $J \pi$-independent decay probability for the exit channel $\chi$. In the context of Surrogate reactions, this approximation greatly simplifies the application of the method: It becomes straightforward to obtain the $J \pi$-independent branching ratios $\mathscr{G}_{\chi}^{C N}\left(E_{e x}\right)$ from measurements of $P_{\delta \chi}\left(E_{e x}\right)\left[=\mathscr{G}_{\chi}^{C N}\left(E_{e x}\right)\right.$ since $\left.\sum_{J, \pi} F_{\delta}^{C N}\left(E_{e x}, J, \pi\right)=1\right]$ and to calculate the desired reaction cross section. Calculating the direct-reaction probabilities $F_{\delta}^{C N}\left(E_{e x}, J, \pi\right)$ and modeling the decay of the compound nucleus are no longer required.

The early applications of the Surrogate approach made use of the Weisskopf-Ewing approximation: The transfer reactions of the 1970s $[4,5]$ produced $(n, f)$ cross section estimates for various actinide targets which agreed with direct measurements (where available) to about 10-20\% for incident neutron energies above $1 \mathrm{MeV}$. Discrepancies at lower energies were attributed to large uncertainties in the low-energy optical model employed, and the use of the Weisskopf-Ewing approximation. More recently, the CENBG collaboration carried out Surrogate experiments to determine cross sections for neutroninduced reactions on several minor actinide nuclei relevant to the thorium-uranium fuel cycle and the transmutation of nuclear waste $[9,12,25]$. The transfer reactions ${ }^{232} \mathrm{Th}\left({ }^{3} \mathrm{He}, \mathrm{x}\right)$ and ${ }^{243} \mathrm{Am}\left({ }^{3} \mathrm{He}, \mathrm{x}\right)$, with $\mathrm{x}=\alpha, \mathrm{t}, \mathrm{d}, \mathrm{p}$, were employed to obtain $(\mathrm{n}, \mathrm{f})$ and $(\mathrm{n}, \gamma)$ cross sections for Th and $\mathrm{Pa}$ targets $[9,12]$ and (n,f) cross sections for $\mathrm{Cm}$ and Am targets [25], respectively. The STARS/LiBerACE collaboration employed the Weisskopf-Ewing approximation to determine the ${ }^{237} \mathrm{~Np}(\mathrm{n}, \mathrm{f})$ cross section, for neutron energies between 10 and $20 \mathrm{MeV}$, from a Surrogate measurement of the ${ }^{238} \mathrm{U}\left({ }^{3} \mathrm{He}, \mathrm{t}\right)$ charge exchange reaction [26]. The cross sections obtained from a WeisskopfEwing analysis of these Surrogate experiments agree reasonably well with directly-measured cross sections.

\section{Ratio approximation}

The Surrogate Ratio approach [10, 14, 15] is an approximation that makes use of the Surrogate idea and requires the (approximate) validity of the Weisskopf-Ewing limit. In this approach, the ratio $R(E)=\sigma_{\alpha_{1} \chi_{1}} / \sigma_{\alpha_{2} \chi_{2}}$ of the cross sections of two compound-nuclear reactions is measured, using two Surrogate experiments. An independent determination of the cross section $\sigma_{\alpha_{1} \chi_{1}}$ can then be used to deduce $\sigma_{\alpha_{2} \chi_{2}}$. An advantage of using the Ratio method is the fact that it eliminates the need to accurately measure the total number of Surrogate reaction events.

The Ratio method has been employed to estimate cross 
sections for neutron-induced reactions on the unstable nucleus ${ }^{237} \mathrm{U}$. Inelastic deuteron [10] and $\alpha$ [14] scattering experiments on ${ }^{238} \mathrm{U}$ and ${ }^{236} \mathrm{U}$ were carried out to obtain the fission probabilities for ${ }^{238} \mathrm{U}$ relative to those for ${ }^{236} \mathrm{U}$. The measured ratio of fission probabilities was then related to the ratio $\sigma\left[{ }^{237} \mathrm{U}(\mathrm{n}, \mathrm{f})\right] / \sigma\left[{ }^{235} \mathrm{U}(\mathrm{n}, \mathrm{f})\right]$ and the desired ${ }^{237} U(n, f)$ cross section was determined with the help of the known ${ }^{235} \mathrm{U}(\mathrm{n}, \mathrm{f})$ cross section. The analysis [14] resulted in a cross section for the ${ }^{237} \mathrm{U}(\mathrm{n}, \mathrm{f})$ reaction that was found to be in good agreement with an earlier theoretical estimate by Younes et al. [8].

\section{Validity of the approximations}

The use of the Weisskopf-Ewing or Ratio approximations in the analysis of Surrogate experiments is typically justified a posteriori by comparing the extracted cross sections to direct measurement where available. A few experiments have been specifically designed to test the approximations: Lesher et al. [27] carried out an inelastic scattering experiment with $\alpha$ particles on ${ }^{234} \mathrm{U}$ and ${ }^{236} \mathrm{U}$ to test the Ratio approach. Good agreement of the extracted ${ }^{235} U(n, f)$ cross section with the direct measurement was found. Lyles et al. [28] focused on possible spin effects in $\left({ }^{3} \mathrm{He}, \alpha\right)$ Surrogate reactions on ${ }^{235,238} \mathrm{U}$. They determined the ${ }^{236} \mathrm{U}(\mathrm{n}, \mathrm{f})$ cross section, employing both the Weisskopf-Ewing and Ratio approximations. The Ratio method led to good agreement with the expected results for neutron energies above about $3 \mathrm{MeV}$ and discrepancies below that. The Weisskopf-Ewing approach could not be used for energies above about 4-5 $\mathrm{MeV}$, due to target contaminants. At neutron energies below about $1.5 \mathrm{MeV}$, a clear indication of spin effects was observed: While the extracted cross section agrees reasonably well with the direct measurements when all $\alpha$-fission coincidence events measured in the Surrogate experiment are taken into account, the cross section was found to depend on the angle of the outgoing $\alpha$ particle when narrower angular ranges are considered. Since the angle of the outgoing particle determines the angularmomentum transfer between projectile and target, and thus the spin-parity distribution of the intermediate system, the findings indicate deviations from the WeisskopfEwing limit.

Recently, calculations were carried out to test the assumptions underlying the Weisskopf-Ewing approximation for fission of uranium nuclei [15]. Individual fission probabilities were extracted from a full Hauser-Feshbach simulation of $(\mathrm{n}, \mathrm{f})$ reactions, and their dependence on $J \pi$ and energy was investigated. The fission probabilities were found to exhibit a significant dependence on angular momentum and a weak dependence on parity, in particular for neutron energies below about $3 \mathrm{MeV}$ and at the onset of second-chance fission. Assuming some reasonable Surrogate $J \pi$ distributions $F_{\delta}^{C N}\left(E_{e x}, J, \pi\right)$ and combining these with the calculated fission probabilities $G_{\chi}^{C N}\left(E_{e x}, J, \pi\right)$, one can simulate observables that are measured in a Surrogate experiment and carry out an analysis of the simulated result invoking the WeisskopfEwing approximation. For the ${ }^{235} \mathrm{U}(\mathrm{n}, \mathrm{f})$ case, e.g., the resulting fission cross section was found to be in rough agreement with the expected result, with the largest deviations occurring for energies below about $3 \mathrm{MeV}$. The study demonstrated that the validity of the WeisskopfEwing approximation depends not only on the energy range considered but also on the $J \pi$ values of the states that are populated in the reaction under consideration. It also illustrated that the cross section estimates can be improved by using the Surrogate Ratio method. Although the Ratio method is based on the assumption that the Weisskopf-Ewing approximation is valid, the simulations indicated that small to moderate deviations from this assumptions might cancel in this approach, thus improving the agreement with the expected results.

$(n, \gamma)$ reactions are expected to be more sensitive to the compound-nucleus spin-parity distribution than $(n, f)$ reactions. Surrogate experiments that aim at determining $(n, \gamma)$ cross sections have to detect the outgoing directreaction particle in coincidence with an observable that identifies the $\gamma$ emission decay channel; for an even-even compound nucleus this is typically a characteristic $\gamma$ ray. In Ref. [11], it was shown that $\gamma$-ray yields from a decaying uranium nucleus depend sensitively on the spin distribution present in the compound nucleus prior to the decay. This sensitivity is even more extreme in nuclei near closed shells: The possibility of determining the ${ }^{91} \operatorname{Zr}(\mathrm{n}, \gamma)$ cross section from a Surrogate experiment was investigated by Forssén et al. [29]. For neutron energies below about $2.5 \mathrm{MeV}$, the gamma decay probabilities were found to depend very sensitively on angular momentum and parity, due to the low level densities in the region and the fact that the compound nucleus can decay can only by gamma or neutron emission. Thus, it is not clear whether the Weisskopf-Ewing approximation can be employed to determine $(n, \gamma)$ cross sections. Experiments that explore the use of the Ratio approach for $(n, \gamma)$ reactions on rare earth nuclei are underway $[30,31]$.

\section{CONCLUSIONS}

Surrogate experiments carried out so far illustrate both the potential and the current limitations of the method. The analyses of present-day experiments, which rely on approximations, yield cross sections that are in reasonable agreement with expected results, for a limited range of applications. As new areas of application are explored, it becomes necessary to move beyond the approximate 
approaches. A comprehensive theoretical treatment of the Surrogate approach involves a description of direct reactions that populate highly-excited, unbound states, the damping of these doorway states into more complicated configurations that lead to a compound nucleus (or non-equilibrium particle emission), the dependence and influence of these processes on angular momentum, parity, and energy, and possible width fluctuation corrections to the standard Hauser-Feshbach-type formalism. Research efforts in this area will contribute to a more fundamental understanding of nuclear reaction mechanisms and help establish an indirect method for obtaining for cross sections that cannot be measured.

\section{ACKNOWLEDGMENTS}

I wish to express my gratitude to Frank Dietrich for fruitful and inspiring discussions throughout the work on this project. This work was performed under the auspices of the U.S. Department of Energy by Lawrence Livermore National Laboratory (LLNL) in part under contract W-7405-Eng-48 and in part under contract DE-AC5207NA27344.

\section{REFERENCES}

1. H. Weidenmüller, "Statistical Theory of CompoundNuclear Reactions," in these proceedings.

2. E. Gadioli and P. E. Hodgson, Pre-Equilibrium Nuclear Reactions, Clarendon Press, Oxford, 1992.

3. H. Weidenmüller, "Precompound Reactions: Basic Concepts," in these proceedings.

4. J. D. Cramer and H. C. Britt, Nucl. Sci. and Eng. 41, 177 (1970).

5. H. C. Britt and J. B. Wilhelmy, Nucl. Sci. and Eng. 72, 222 (1979).

6. W. Younes and H. C. Britt, Phys. Rev. C 67, 024610 (2003).

7. W. Younes and H. C. Britt, Phys. Rev. C 68, 034610 (2003).

8. W. Younes, H. C. Britt, J. A. Becker, and J. B. Wilhelmy, Initial estimate for the ${ }^{237} \mathrm{U}(\mathrm{n}, \mathrm{f})$ cross section for $0.1 \leq$ $E_{n}(\mathrm{MeV}) \leq 20$, Tech. Rep. UCRL-ID-154194, Lawrence Livermore National Laboratory, Livermore, CA (2003).

9. M. Petit, M. Aiche, G. Barreau, et al., Nucl. Phys. A 735, 347-371 (2004).

10. C. Plettner, et al., Phys. Rev. C 71, 051602(R) (2005).

11. J. E. Escher and F. S. Dietrich, Examination of the validity of the Surrogate Ratio Method for determining $(\mathrm{n}, \mathrm{f})$ and $(\mathrm{n}, \gamma)$ cross sections of actinides, Tech. Rep. UCRL-TR-212509-Draft, Lawrence Livermore National Laboratory, Livermore, CA (2005).

12. S. Boyer, D. Dassie, J. Wilson, M. Aiche, G. Barreau, S. Czajkowski, C. Grosjean, A. Guiral, B. Haas, B. Osmanov, G. Aerts, E. Berthoumieux, F. Gunsing, C. Theisen, N. Thiolliere, and L. Perrot, Nucl. Phys. A 775, 175-187 (2006).
13. V. M. Maslov, Phys. Rev. C 72, 44607 (2005).

14. J. Burke, L. Bernstein, J. Escher, et al., Phys. Rev. C 73, 054604 (2006).

15. J. E. Escher and F. S. Dietrich, Phys. Rev. C 74, 054601 (2006).

16. J. Escher, F. S. Dietrich, and C. Forssén, Nucl. Instr. and Meth. B 261, 1075 (2007).

17. J. Escher, L. A. Bernstein, J. T. Burke, F. S. Dietrich, C. Forssén, and B. Lyles, "Surrogate Reactions: The Weisskopf-Ewing Approximation and its Limitations," in International Conference on Nuclear Data for Science and Technology (ND2007), Nice, France, April 22-27, 2007, (to be published).

18. W. Hauser and H. Feshbach, Phys. Rev. 87, 366-373 (1952).

19. B. L. Andersen, B. B. Back, and J. M. Bang, Nucl. Phys. A 147, 33-44 (1970).

20. B. B. Back, O. Hansen, H. C. Britt, and J. D. Garrett, Phys. Rev. C 9, 1924-1947 (1974).

21. J. Escher and F.S. Dietrich, Producing a compound nucleus via a inelastic scattering: The ${ }^{90} \mathrm{Zr}\left(\alpha, \alpha^{\prime}\right)^{90} \mathrm{Zr}^{*}$ case, Tech. Rep., Lawrence Livermore National Laboratory, Livermore, CA (2008), in preparation.

22. F. Dietrich, "Compound-Nucleus Formation Following Direct Interactions to Highly-Excited Final States," in these proceedings.

23. W. E. Parker, M. B. Chadwick, F. S. Dietrich, J. E. Kammeraad, S. J. Luke, K. E. Sale, R. M. Chasteler, M. A. Godwin, L. H. Kramer, G. J. Schmid, H. R. Weller, and A. K. Kerman, Phys. Rev. C 52, 252 (1995).

24. F. S. Dietrich, Simple derivation of the Hauser-Feshbach and Weisskopf-Ewing formulae, with application to Surrogate reactions, Tech. Rep. UCRL-TR-201718, Lawrence Livermore National Laboratory, Livermore, CA (2004).

25. B. Jurado, "Fission Cross Sections and Fission-Fragment Mass Yields via the Surrogate Reaction Method," in these proceedings.

26. M. Basunia, et al., "Study of the $\left({ }^{3} \mathrm{He}, \mathrm{t}\right)$ Charge Transfer Reaction as a Surrogate for Neutron Energy Between 10 to $20 \mathrm{MeV}$," in these proceedings.

27. S. Lesher, et al., "Benchmarking the External Surrogate Ratio Method using the $\left(\alpha, \alpha^{\prime} f\right)$ reaction at STARS," in these proceedings.

28. B. F. Lyles, L. A. Bernstein, J. T. Burke, F. S. Dietrich, J. Escher, I. Thompson, D. L. Bleuel, R. M. Clark, P. Fallon, J. Gibelin, A. O. Macchiavelli, M. A. McMahan, L. Phair, E. Rodriguez-Vieitez, M. Wiedeking, C. W. Beausang, S. R. Lesher, B. Darakchieva, and M. Evtimova, Physical Review C 76, 014606 (2007).

29. C. Forssén, F. Dietrich, J. Escher, R. Hoffman, and K. Kelley, Phys. Rev. C 75, 055807 (2007).

30. R. Hatarik, et al., "Is (d,p $\gamma)$ a surrogate for neutron capture?," in these proceedings.

31. N. Scielzo, et al., "Determininig the $(\mathrm{n}, \gamma)$ cross section of ${ }^{153} \mathrm{Gd}$ using surrogate reactions," in these proceedings. 